\title{
CURVA DE CRESCIMENTO DE CODORNAS DE CORTE POR MEIO DE MODELOS DE REGRESSÃO NÃO-LINEARES
}

\author{
(Meat quail growth curve by non-linear regression models)
}

Mérik Rocha-Silva, Claudio Vieira Araujo, Aldrin Vieira Pires, Edson Junior Heitor DE PAULA, Eric Batista Ferreira, Felipe Gomes DA SILVA ${ }^{1}$

${ }^{1}$ Correspondência: merikrocha@hotmail.com

RESUMO: Objetivou-se avaliar diferentes modelos de regressão não-linear para descrição da curva de crescimento de codornas de corte, machos e fêmeas. Foram utilizados 30.410 registros de peso corporais, originalmente mensurados semanalmente de codornas de corte, do $1^{\circ}$ ao $42^{\circ}$ dia de idade, participantes do Programa de Melhoramento Genético da Universidade Federal dos Vales do Jequitinhonha e Mucuri. O peso corporal das aves foram agrupados em machos ou fêmeas e submetidos aos modelos de regressão não-lineares de Brody, Gompertz, Logístico, MMF (Morgan-Mercer-Flodin), Richards, e van Bertalanffy. Os modelos foram avaliados quanto ao ajuste das curvas de crescimento preditas em relação aos valores observados. Não se obteve convergência com o modelo Brody. Os demais modelos apresentaram bons valores de IA (índice assintótico), sendo o modelo MMF o mais adequado para predizer o peso dos machos com IA 33,59, enquanto que o modelo Gompertz com IA 36,100, apresentou-se como mais adequados para predizer o peso das fêmeas. Portanto, no presente estudo os modelos de Gompertz e MMF apresentaram melhor ajuste aos dados para machos e para fêmeas; com $\mathrm{R}^{2}$ aj acima de 0,92 . Os resultados indicam a utilização de ambos modelos para descrever o crescimento dos animais, independente do sexo.

Palavras-chave: Coturnix coturnix, Nlin, Gompertz, MMF Morgan-Mercer-Flodin

ABSTRACT: The aim of this study was to evaluate different non-linear regression models to describe the growth curve of male and female meat quail. The study involved 30,410 records of body weight originally measured weekly from the 1st to the 42nd day of life of meat quail participating in the Breeding Program of the Federal University of Vales do Jequitinhonha e Mucuri. Body weight was separated into the male and female classes and subjected to the non-linear Brody, Gompertz, Logistic, MMF (Morgan-Mercer-Flodin), Richards, and van Bertalanffy models. The models were evaluated with respect to the adjustment of predicted growth curves in relation to observed values. No convergence was obtained with the Brody model. The other models yielded good Al (asymptotic index) values, and the MMF model was the most suitable to predict the weight of males, with an Al of 33.59, whereas the Gompertz model, with an Al of 36.100 , was the most suitable to predict the weight of females. Therefore, in the present study, the Gompertz and MMF models best fitted the data for males and females, with an R2aj greater than 0.92. Results point to the use of both methods to describe the growth of these animals, irrespective of sex.

Key Words: Coturnix coturnix, Nlin, Gompertz, MMF Morgan-Mercer-Flodin 


\section{INTRODUÇÃO}

O Brasil está consolidado como um dos principais produtores avícolas há alguns anos, todavia, a coturnicultura ainda têm muito o que ser desenvolvida, sendo que pelo menos até 2002 não havia no Brasil programas de melhoramento de codornas baseado em normas técnicas, sejam de postura ou de corte.

Essa deficiência gerou sérios prejuízos à coturnicultura, principalmente relacionados à consanguinidade, repercutindo em redução da postura, queda da fertilidade e aumento da mortalidade (MARTINS, 2002). Caracterizando a necessidade, entre outros, do desenvolvimento de técnicas e metodologias com vistas ao melhoramento genético, e se cabível o desenvolvimento de linhagens melhores, seja para maior precocidade no ganho de peso, seja para outras características de interesse econômico.

A principal característica fenotípica de interesse comercial na coturnicultura de corte é o peso corporal; que possui de média a alta herdabilidade, sendo passível de ser utilizado como critério para seleção de animais mais precoces entre linhagens ou entre animais da mesma linhagem, principalmente durante a fase de crescimento (KNÍZETOVÁ et al., 1995).

Os modelos não-lineares são empregados para descrever a curva de crescimento de animais de produção, para interpretação do desenvolvimento de diversas espécies domésticas: bovinos, suínos, frangos, ovinos, coelhos, codornas, entre outros (NAHASHON et al., 2005; FREITAS, 2005).

O modelo de regressão nãolinear de von Bertalanffy, segundo Richards (1959), foi o primeiro a ser empregado para o estudo de curvas de crescimento. As re-parametrizações deste deram origem aos modelos de Brody, van Bertalanffy, Richards, entre outros. A partir do modelo de Richards deu-se adaptações que culminaram com os modelos Logístico e de Gompertz (NAHASHON et al., 2006).

Pode-se abreviar uma série de informações repetidas de qualquer animal ou grupo de indivíduos, sob diferentes tratamentos (sexo, raça/linhagem, dieta, etc.) submetidos sob diferentes condições (tempo, níveis, doses, etc.) em três ou quatro parâmetros de um destes modelos de não lineares (FREITAS, 2005).

Assim, promove-se

interpretação de várias pesagens em três ou quatro parâmetros, estimando-se os efeitos individualizados por cada linhagem ou sexo; e principalmente, os animais mais precoces para peso, gerando informações fomentadoras de seleção de animais de genética superior para precocidade.

Estes modelos têm sido usados também para relacionar o peso corporal das aves (e outros animais) com a ingestão cumulativa, determinar a eficiência de utilização de nutrientes; bem como com funções para prever os requisitos de energia e proteína visando a manutenção do crescimento (DARMANI-KUHI et al., 2010)

Potencialmente pode-se empregar qualquer um dos seis modelos matemáticos utilizados neste estudo (Brody, Gompertz, Logístico, MMF (Morgan-Mercer-Flodin), Richards, e van Bertalanffy), todavia, há variação suficiente entre espécies e até mesmo entre linhagens, inclusive entre indivíduos da mesma linhagem; que projetam haver um modelo mais apropriado a cada conjunto de dados fenotípicos. As pesquisas já realizadas com os modelos Brody, von Bertalanffy, Logístico e Gompertz (FIGUEIREDO et al., 2010) e o modelo de Richards (DRUMOND et al., 2013), modelos hiperbólicos (NARINC et al., 2010); demonstram que há observações a serem feitas e estudos para elucidar 
com precisão o modelo mais ajustado em cada caso.

O objetivo com este trabalho foi identificar o modelo de regressão não linear mais adequado para descrição do crescimento corporal em função da idade, em codornas de corte, agrupadas por sexo.

\section{MATERIAL E MÉTODOS}

\section{Os animais}

Foram utilizados 6.797 codornas de corte pertencentes ao Programa de Melhoramento de Codornas da Universidade Federal dos Vales do Jequitinhonha e Mucuri (UFVJM) em Diamantina, MG.

Após análise dos dados; procedeu-se a eliminação de outliers improváveis e as informações de animais sem dados em todas as pesagens; culminando em 30.410 registros de peso corporal de codornas de corte (Coturnix coturnix) da linhagem LF1 oriundos das pesagens semanais, do nascimento até a idade de abate (1 a 42 dias).

Os

animais

foram majoritariamente mantidos em boxe com piso de concreto, cama de maravalha, com aquecimento via campânulas. Estes foram alimentados segundo duas formulações: até os 21 dias, dieta apresentando em média $25 \%$ de proteína bruta e $2900 \mathrm{kcal}$ de $\mathrm{EM} \mathrm{kg}{ }^{-1}$, sequencialmente até $042^{\circ}$ dia dieta mais calórica e menos proteica, 24\% PB e $2925 \mathrm{kcal}^{\mathrm{de}} \mathrm{EM} \mathrm{\textrm {kg } ^ { - 1 }}$ segundo recomendações de exigências nutricionais da literatura nacional (OLIVEIRA et al., 2002; VELOSO et al., 2012).

\section{Modelos de Regressão Não Linear}

O peso corporal das aves foi previamente ajustado para o efeito de data de eclosão, por meio das soluções de quadrados mínimos.
A partir de dados observados ao longo do tempo deu-se a análise das variáveis quantitativas empregando-se os modelos matemáticos dispostos na Tabela 1, para descrever o crescimento, a partir do ajuste destes modelos pela estimação dos parâmetros.

Tabela 1- Parametricaça dos modelos analsados.

\begin{tabular}{|c|c|}
\hline Modelos & Equapoes \\
\hline Brody (5ARMENTO et al., 2006) & $Y_{1}=A\left(1-B e^{\prime \prime}\right)+?$ \\
\hline Gompertz (FLALHO, 1969) & $Y_{i}=A e+\pi M+?$ \\
\hline Logistico (O et al.(2012) & $Y_{1}=A\left(1+B e^{-5}\right)^{1}+?$ \\
\hline Mogan-Mercer-Flcan (NAFINC ef al:(2010) & $\gamma_{1}=\left\langle A B+k t^{d}\right\rangle\left\langle\left(B+1^{t}\right\rangle+?\right.$ \\
\hline Richards (SARAENTO et al, 2006) & $\gamma_{1}=A\left(1-B e^{k y}+\right.$ ? \\
\hline won Bertzanfly (DRUMCND et al .2013) & $Y_{1}=A\left(1-B e^{n}\right)^{3}+?$ \\
\hline
\end{tabular}
curva

Nos modelos as mudanças no tamanho são representadas por $Y$ (peso corporal) em relação à idade $t$ (tempo em dias de vida). O parâmetro $A$, também podendo ser denominado $B_{0}$ ou até mesmo $B_{1}$ por alguns autores, sempre representando em modelos de regressão não linear o peso corporal assintótico ou peso corporal a maturidade, parâmetro de característica fenotípica (MCMANUS et al., 2003; DRUMOND et al., 2013).

O parâmetro $B$, é uma constante de integração. Este parâmetro indica a proporção do crescimento assintótico a ser ganho depois do nascimento, sem interpretação biológica clara (MCMANUS et al., 2003; DRUMOND et al., 2013).

$\mathrm{O}$ índice de maturidade $\mathrm{K}$ ou B3 é uma medida de precocidade; possibilitandonos aferir o tempo empregado pelos animais para atingir a maturidade; assim sendo, animais mais precoces quanto à maturidade apresentam $k$ maiores (MCMANUS et al., 2003; DRUMOND et al., 2013).

O parâmetro $m$ não é comum a todos os modelos. Este indica o ponto de inflexão, dando principalmente forma à curva. Neste ponto é indicado quando o crescimento do animal passa de acelerado para um crescimento inibitório ou reduzido, associado principalmente a fatores hormonais alterados pelo estágio 
fisiológico da puberdade dos animais (MCMANUS et al., 2003; DRUMOND et al., 2013).

Utilizando o procedure NLIN do SAS ${ }^{\circledR}$ 9.2 estimaram-se os parâmetros dos modelos pelo algoritmo de Gauss Newton (HARTLEY, 1961); utilizando-se o método dos quadrados mínimos.

Calculou-se o coeficiente de determinação ajustado $\left(\mathrm{R}^{{ }^{2} \mathrm{ij}}\right)$ como sendo o coeficiente de determinação entre a o valor do peso corporal regredido em função de sua predição, para ser empregado na avaliação do melhor modelo.

Para tais comandos auxiliares do software SAS foram empregados; o proc reg para obtenção do coeficiente de regressão; proc means; var para obtenção da soma de quadrados do resíduo empregado no cálculo do Desvio Médio Absoluto (DMA). Para obtenção da soma de quadrado médio do erro foram indicados o $\mathrm{N}$ (número de amostras) para o sexo 1 (machos) 15002 e para o sexo 2 (fêmeas) 15408.

Para indicação do melhor modelo, verificou-se qual apresentou menor Índice Assintótico (IA), descrito na Equação 3. O Desvio Padrão Assintótico (DPA), Equação 1, é obtido a partir da raiz quadrada do quadrado médio do resíduo de cada modelo, e o Desvio Médio Absoluto (DMA) dos resíduos (Equação 2) conforme proposto por Henriques et al. (2011) e Sarmento et al. (2011).

Equação 1 - Desvio Padrão Assintótico

$D P A=\sqrt{\frac{\sum_{i}^{1}\left(Y_{i}-\hat{Y}_{i}\right)}{n}}$

Equação 2 - Desvio Médio Absoluto

$D M A=\frac{\sum_{i}^{1}\left|Y_{i}-\hat{Y}_{i}\right|}{n}$

Equação 3 - Îndice Assintótico

$I A=(D P A+D M A)-R^{2}$

$Y_{j}$ é o valor observado $\hat{Y}_{j}$ o valor estimado e $n$ o tamanho da amostra.
A estimação do Critério de Informação de Akaike-AIC (Equação 4) e de informação Bayesiana- BIC (Equação 5), foram emitidos utilizando o mesmo software estatístico.

$A I C=-\log L i k e+2 p$, (Equação

Critério de Informação de Akaike)

$B I C=-2 \log l i k e+2 p \ln (\mathrm{n}) \quad, \quad(E q u a c ̧ a ̃ o$

5: Critérios de Informação Bayesiano)

Onde:

pé o número de parâmetros;

$L=$ Likelihood

logLike é o logaritmo da função de verossimilhança.

\section{RESULTADOS E DISCUSSÃO}

Como esperado para esta espécie, o peso corporal médio das fêmeas se manteve-se superior aos dos machos em todas as idades, diferença acentuada com o avançar da idade, principalmente em decorrência do desenvolvimento do aparelho reprodutivo e maior taxa de deposição de gordura (DRUMOND et al., 2013). Em média 0 aparelho reprodutor representa $10 \%$ do peso corporal vivo de uma codorna adulta (ALBINO e BARRETO, 2003). Em ambos os sexos os desvios padrão foram maiores aos 28 dias, (Tabela 2).

\begin{tabular}{|c|c|c|c|c|c|c|}
\hline \multirow{2}{*}{$\begin{array}{l}\text { Idade } \\
\text { (dias) }\end{array}$} & \multicolumn{3}{|c|}{ Machos $(g)$} & \multicolumn{3}{|c|}{ Femeas (g) } \\
\hline & N & Média & $D P$ & N & Mecia & $D P$ \\
\hline 1 & 2702 & 9,18 & 0,99 & 2782 & 9,22 & 1,08 \\
\hline 7 & $24 \% 3$ & 28,14 & 6,41 & 2486 & 28,57 & 657 \\
\hline 14 & $245 t$ & 66,61 & 17,05 & 2504 & 67,68 & 18.08 \\
\hline 21 & 2455 & 113,47 & 27,85 & 2526 & 115,81 & 30,53 \\
\hline 28 & 2492 & 164,11 & 32,25 & 2589 & 169,36 & 3523 \\
\hline 35 & 2469 & 211.51 & 30,35 & 2541 & 219.58 & 33.10 \\
\hline 42 & 1522 & 238,16 & 28,46 & 1555 & 248,66 & 32,71 \\
\hline
\end{tabular}

Observa-se intenso crescimento inicial, principalmente durante a primeira semana, quando os animais apresentam ganho de peso semanal superior a $200 \%$. Apesar de o crescimento ter se mantido constante, as taxas são menos intensas após este período. 
As estimativas que compõem a Tabela 3 demonstram expressiva variação entre os modelos, sendo identificado que 0 peso corporal à maturidade $(\hat{A})$ foram superestimados.

Denota-se que o ganho de peso predito pelo modelo de Gompertz emprega como parâmetro um peso assintótico de $369 \mathrm{~g}$ para codornas de corte machos e $404 \mathrm{~g}$ para codornas de corte fêmeas. Todavia, quando o modelo de Gompertz é efetivamente empregado para predizer os pesos aos 42 dias, prediz peso corporal em torno de 254 e $268 \mathrm{~g}$, mais próximos dos pesos corporais observados. Ou seja, o peso corporal assintótico creditado ao valor do parâmetro $A$ pelo modelo não perfaz exatamente o peso do animal adulto, mas sim uma estimação de quando o animal será adulto, o que ocorre após os 42 dias.

Tabela 3 - Estimatvas dos parabmetros das curvas de crescimento para codomas de corte. machos efémeas. para diferentes modelos.

\begin{tabular}{|c|c|c|c|c|}
\hline \multirow{2}{*}{ Modelo } & \multicolumn{4}{|c|}{ Estimativas } \\
\hline & $y^{n}$ & $\mathrm{~B}^{2}$ & $7 n^{n}$ & $\pi / m^{4}$ \\
\hline \multicolumn{5}{|c|}{ - Machos - } \\
\hline Gompertz & 369,2 & $-0,0533$ & 23,5832 & - \\
\hline Logistion & 281.1 & 15,9859 & 0,12 & - \\
\hline Morgan Mercer Fiodn & 676,2 & 0.00122 & 12,4660 & $-1,6559$ \\
\hline Richards & 393.2 & 0.2811 & 0.0477 & 12.5022 \\
\hline van Bertalanffy & 635.0 & 0,7427 & 0,0298 & $\cdot$ \\
\hline \multicolumn{5}{|c|}{-Fatmeas - } \\
\hline Gomperts & 404,1 & $-0,0510$ & 24,5678 & - \\
\hline Logistice & $2 \pi 7.8$ & 15,8406 & 0,11 & - \\
\hline Morgan Mercer Flodin & 735,1 & 0.00110 & 14,1380 & $-1,6684$ \\
\hline van Bertalanffy & 810,1 & 0,7432 & 0.0276 & - \\
\hline
\end{tabular}

O modelo de Gompertz indicou o ponto de inflexão acima de 23 e 24 dias, respectivamente para machos e fêmeas. Segundo Cecconello et al. (2009), podese balizar pelo ponto de inflexão para determinar dentro da linha de tempo o momento do abate dos animais, sendo por vezes, a ocasião de aproveitamento ótimo da produção com interesse em carne.

O modelo de Brody não apresentou convergência para os dados estudados. Modelos com menor nível de ajustamento repercutem em superestimação ou subestimação na predição de peso. A partir do exposto pelas figuras abaixo observa-se relativa precisão dos modelos para predizer 0 peso nas diferentes idades em cada sexo. $O$ modelo de Gompertz superestimou todos os pesos corporais de animais de ambos os sexos, principalmente nas pesagens finais $35 \mathrm{e}$ 42 dias (Figura 1).

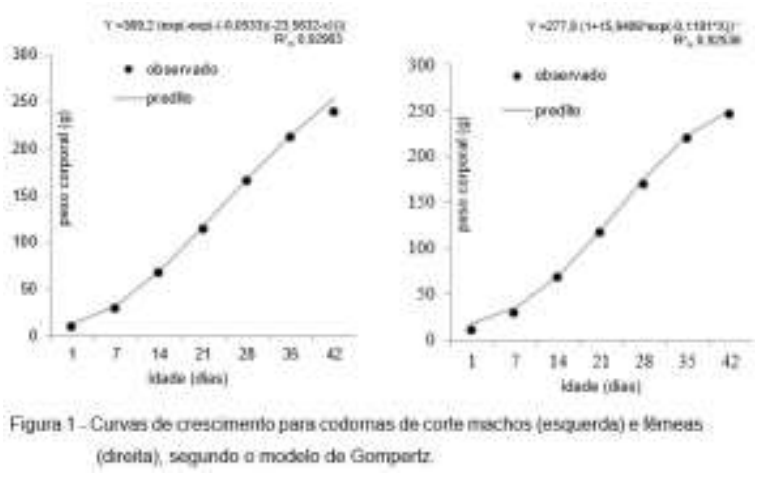

O modelo logístico também superestimou os pesos corporais das codornas fêmeas, no entanto, promoveu algumas subestimações sutis para os pesos corporais aos 14, 35 e 42 dias de vida dos machos (Figura 2).
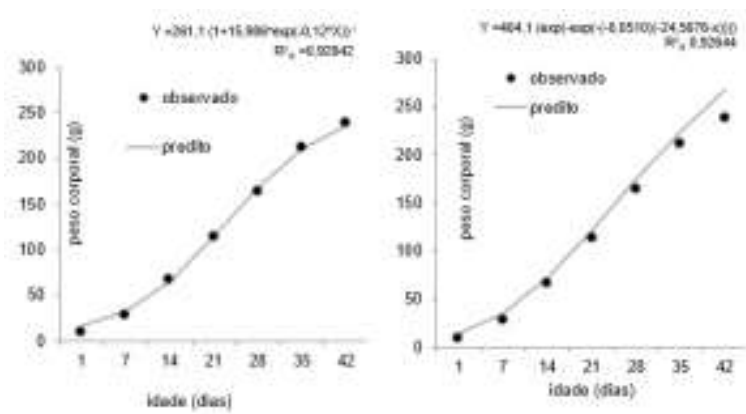

Figure 2-Curvas ib crescimento pera codamas de corte machos (esquarda) e sameas (divita), Stagundo o modelo Logistico.

O modelo de Morgan-MercerFlodin (MMF) inversamente ao logístico e assim como Gompertz superestimou os pesos corporais dos machos. Subestimou o peso dos animais fêmeas aos 7 dias (Figura 3). 


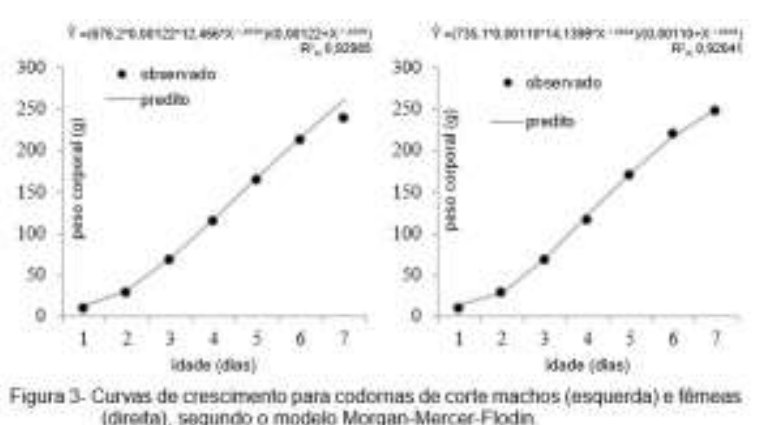

O modelo de von Bertalanffy (Figura 4) e Richards (Figura 5) também superestimaram os pesos corporais que predisseram. Assim como nos experimentos de Drumond et al. (2013), que identificaram superestimação do peso assintótico pelo modelo de von Bertalanffy; repercutindo em menor estimativa de taxa de maturidade (“k”), principalmente em decorrência de ter sido identificado pelos autores correlação fenotípica negativa de alta magnitude entre os parâmetros "A" e " $k$ " (e positiva entre "m" e "k").
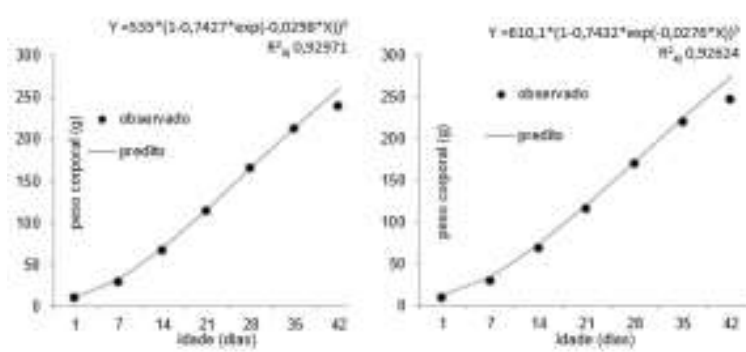

Fora 4 - Curvas de crescinemo para cosomas de cone machos (esqueda) e Smeas. (drena), segundo a modaib von Bortalanity

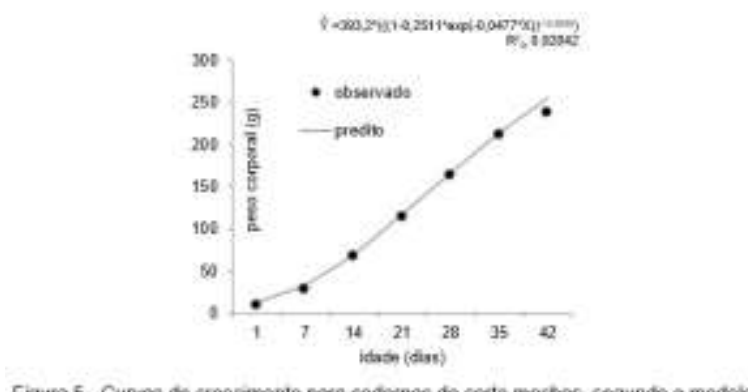

Figura 5 - Curvas de crescimento para codoctias de corte machos, segundo a modelo Fichords

As estimativas de $R^{2}$ aj para os modelos que convergiram (Tabela 4) são adequados para aferir os modelos como suficientemente capazes de justificar a maior parte da variação $\left(R^{2}{ }_{a j}\right.$
$>0,925)$. O que em concordância com Hasan-Eleroglu et al. (2014) já é suficiente para validar 0 uso dos modelos.

Segundo Drumond et al. (2013), o $\mathrm{R}^{2}$ isoladamente pode ser empregado como critério, no entanto, em seus estudos, apresentou valores altos $\mathrm{e}$ semelhantes; justificando o uso do IA. O de acordo com Eleroğlu et al.(2014) já é suficiente como validador do uso dos modelos. Por fim, os supracitados autores concluíram que os animais que atingem a maturidade mais pesados apresentaram menor taxa de crescimento; e que quanto maior a inclinação da curvatura das funções, tenderá ser maior a precocidade dos animais.

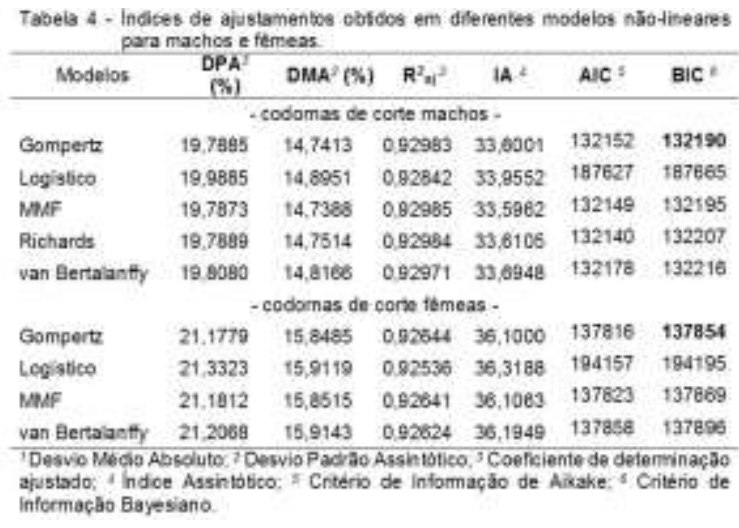

Notoriamente dois modelos se destacaram como mais ajustados para predizer os pesos corporais dos animais machos e fêmeas: Gompertz e MMF. O modelo desenvolvido por Morgan, Mercer e Flodin (1975) superou os demais, com exceção de Gompertz.

Entre os modelos que apresentaram convergência, o Índice Assintótico (IA) sequencia os mais ajustados, por ordem: MMF $(33,59)$, Gompertz $(33,60)$, Richards $(33,61)$, van Bertalanffy $(33,69)$ e Logístico $(33,95)$ para os animais machos. Em relação aos dados das fêmeas, o modelo Gompertz apresentou-se como mais ajustado, superando o modelo MMF, os demais mantiveram suas posições. 
Mais intensamente têm sido empregado os critérios de informação de Akaike (AIC) e Bayseiano (BIC) para aferir e classificar os modelos de crescimento quanto ao ajuste.

Assim, observa-se neste estudo que o IA alterna entre dois modelos. Outrossim, quando considerado BIC como critério obtêm um resultado mais coeso, sendo acusado o modelo Gompertz como mais ajustado independente de sexo. Sequencialmente, a partir dos valores de BIC, os modelos mais ajustados para predizer o peso de codornas de corte machos e fêmeas são: Gompertz, MMF, Richards (não convergiu para pesos das fêmeas), von Bertalanffy e o menos ajustado Logístico.

Semelhantemente, Narinc et al. (2010), em experimento com Coturnix coturnix japonica, obtiveram resultados semelhantes, tendo 0 modelo de Gompertz como o mais ajustado. Sendo MMF mais ajustado que modelos habitualmente utilizados como Logístico, Bertalanffy e Brody.

O terceiro modelo mais ajustados para predizer o ganho de peso corporal de codornas de corte machos, a função de Richards (Richards, 1959) é tida como mais flexível em relação ao modelos Logístico e de Gompertz; sendo capaz de abordar crescimento assimétricos (YIN et al.,2003). Beiki et al. (2013) em seus estudos apontaram o modelo de Richards como mais apropriado para linhagens especificas de codornas de corte, apresentando-se ser mais ajustado que os modelos Hiperbólicos 1, 2 e 3; von Bertalanffy, Logístico.

O modelo de Gompertz pode ser empregado na gestão da atividade através dos conhecimentos das projeções de ganho de peso médio e consequentemente volume de arraçoamento, e principalmente seleção dos animais mais precoces para ganho de peso.
Mariano et al. (2014) avaliando a interação genótipos $X$ ambientes, utilizaram dois genótipos não identificaram diferença significativa dos valores $\mathrm{k}$ e $\mathrm{b}$ entre os animais medianos e de baixo valor genético, somente entre animais de elevado valor genético entre os que compõem as linhagens UFV1 e UFV2 sob análise do modelo de Gompertz. Apontaram em suas conclusões que o parâmetro A (peso médio à maturidade) apresentou-se mais estável à mudança de gradiente nutricional.

\section{CONCLUSÃO}

Cinco dos seis modelos empregados apresentaram convergência com os dados, sendo indicado o uso do modelo de Gompertz para machos e para fêmeas, para descrever a curva de crescimento de codornas.

Considerando as diferenças fisiológicas entre machos e fêmeas, que repercute em pesos distintos, deve-se manter a análise individualizada por sexo, mesmo que o mesmo modelo seja o mais ajustado para ambos os sexos; afinal, os parâmetros estimados são diferentes.

\section{NOTAS INFORMATIVAS}

Reservado ao parecer CEUA.

\section{REFERÊNCIAS}

ALBINO, L.F.T. e BARRETO, SR.L.T. Criação de codornas para produção de ovos e carne. Editora Aprenda Fácil, Viçosa: 2003. 268 p.

BEIKI, H.; PAKDEL, A.; MORADISHAHRBABAK, M. e MEHRBAN, $\mathrm{H}$. Evaluation of Growth Functions on Japanese Quail Lines. J. Poult. Sci., v. 50, 2013. p. 20-27.

CECCONELLO, M.; BRANDÃO, A.J.V.; e BASSANEZI, R.C. Sobre o Ponto de 
inflexão em modelos de crescimento inibido com condição Inicial Fuzzy. Biomatemática v.19; 2009, p. 81-98.

DIONELLO, N.J.L.; CORREA, G.S.S.; SILVA, M.A.; CORREA, A.B.; SANTOS, G.G. Estimativas da trajetória genética do crescimento de codornas de corte utilizando modelos de regressão aleatória. Arq. Bras. Med. Vet. Zootec., v.60, n.2, 2008. p.454-460

DARMANI-KUHI, $\mathrm{H}_{\text {.; }}$ PORTER, T.; LOPEZ, S. et al. A review of mathematical functions for the analysis of growth in poultry. World's Poultry Science Journal, v. 66. 2010. p.227240.

DRUMOND, E.S.C.; GONÇALVES, F.M.; VELOSO, R.C.; et al. Curvas de crescimento para codornas de corte. Ciência Rural, Santa Maria, v.43, n.10. 2013. p.1872-1877.

ELEROGLU, H.; YILDIRIM, A.; SEKEROGLU, A. et al. Comparison of Growth Curves by Growth Models in Slow-Growing Chicken Genotypes Raised the Organic System. Int. J. Agric. Biol., v.16, 2014. p. 529-535.

FREITAS, A.R.; ALBINO, L.F.T; MICHELAN-FILHO, T. et al. Modelos de curva de crescimento em frangos de corte. Pesp. Agrop. Bras. Brasília, v.19, n.9.1984. p.1057-1064.

FIALHO, F.B. Interpretação da curva de crescimento de Gompertz. Comunicado Técnico. EMBRAPA-Empresa Brasileira de Pesquisa Agropecuária - Centro Nacional de Pesquisa de Suínos e Aves, Concórdia, CT 237, 1999. p.1-4

FREITAS, A.R. Curvas de crescimento na produção animal. R. Bras. Zootec.v.34, n.3.2005. p. 786-795.

HARTLEY, H.O. (1961). The modified Gauss-Newton method for the fitting of nonlinear regression functions by least squares. Thechnometrics, 3:269-280.
HENRIQUES, L.T.; VALADARES FILHO, S.C.; FONSECA, M.A. et al. Avaliação de modelos não-lineares e da relação do consumo voluntário de vacas primíparas e de bezerros com a curva de lactação de vacas Nelore. R. Bras. Zootec., v.40, n.6. 2011. p.1287-1295

KNÍZETOVÁ, H.; HYÁNEK, J.; HYÁNKOVÁ, L. et al. Comparative study of growth curves in poultry. Genet Sel Evol. v.27. 1995.p.365-375.

McMANUS, C.; EVANGELISTA, C.; FERNANDES, L.A.C. Curvas de Crescimento de Ovinos Bergamácia Criados no Distrito Federal. R. Bras. Zootec., v.32, n.5, 2003. p.1207-1212,

MARIANO WH, OLIVEIRA LT, PAULA $\mathrm{C}$, et al. Análise genética do crescimento de codornas de corte em diferentes ambientes nutricionais. Anais... XXIV Congresso Brasileiro de Zootecnia. Vitória. 2014.

MARTINS, E.M. Perspectivas do melhoramento genético de codornas no Brasil. Anais do IV Simpósio Nacional de Melhoramento Animal, Campo Grande.2002.

MAZUCHELI, J.; SOUZA, R.M.; e PHILIPPSEN, A.S. Modelo de crescimento de Gompertz na presença de erros normais heterocedásticos: um estudo de caso. Rev. Bras. Biom., São Paulo, v.29, n.1, 2011. p.91-101.

NAHASHON, S.N.; AGGREY, S.E.; ADEFOPE, N.A.; et al. Growth Characteristics of Pearl Gray Guinea Fowl as Predicted by the Richards, Gompertz, and Logistic Models. Poultry Science.v.85. 2006. p. 359-363.

NARINC, D.; KARAMAN, E.; FIRAT, M.Z.; et al. Comparison of non-linear growth models to describe the growth in Japanese quail. Journal of Animal and Veterinary Advances, v.9, n.14: 2010. p. 1961-1966.

Ó, A.O; RÊGO NETO, A.A.; SANTOS, G.V.; SARMENTO, J.L.R.; BIAGIOTTI, 
D.; SOUSA, J.E.R. Curva de crescimento de ovinos Santa Inês no Vale do Gurgueia. Rev. Bras. Saúde Prod. Anim., Salvador, v.13, n.4, p.912922 out./dez., 2012.

OLIVEIRA, E.G.; ALMEIDA, M.I.M.; MENDES, A.A.; VEIGA, N.; DIAS, K. Desempenho produtivo de codornas de ambos os sexos para corte alimentadas com dietas com quatro níveis protéicos. Archives of Veterinary Science, v.7, n.2, p.75-80, 2002.

RAJI, A.O.; MBAP, S.T. e ALIYU, J. Comparison of different models to describe growth of the japanese quail (Coturnix japonica). Trakia Journal of Sciences, v.2. 2014. p.182-188.

RIBEIRO, M.J.B.; Curva de crescimento de codornas ajustada por modelos nãolineares. Dissertação... Mestrado em Zootecnia. Universidade Federal de Sergipe. São Cristóvão: 99.2014

RICHARDS, F.J.A flexible growth function for empirical use. Journal of Experimental Botany, v. 10: 1959. p. 290-300.

SARMENTO, J.L.R.; REGAZZI, A.J.; SOUZA, W.H. et al. Estudo da curva de crescimento de ovinos Santa Inês. R. Bras. Zootec., v.35, n.2, 2006. p. 435442,

SAS Institute Inc. 2009. SAS/STAT User's Guide, Version 9.2. SAS Institute Inc., Cary, NC.

SAS University Edition. SAS release: 9.04.01M1P12042013. SAS platform: Linux LIN X64 2.6.32431.11.2.el6.x86_64. SAS Institute Inc., Cary, NC, USA.

THOLON, P. e QUEIROZ, S.A. Modelos matemáticos utilizados para descrever curvas de crescimento em aves aplicados ao melhoramento genético animal. Ciência Rural, Santa Maria, v.39, n.7, 2009. p.2261-2269.
VELOSO, R.C.; PIRES, A.V.; TIMPANI, V.D.; DRUMOND, E.S.C.; GONÇALVES, F.M.; FARIA FILHO, D. E. Níveis de proteína bruta e energia metabolizável em uma linhagem de codorna de corte. Acta Scientiarum. Animal Sciences, v.34, n.2, p.169-174, 2012.

YIN, X.; GOUDRIAAN, J.; LATINGA, E.A.; et al. A flexible sigmoid function of determinate growth. Annual Botany, n.91. 2003. p. 361-371. 